\title{
New Proofs of Plünnecke-type Estimates for Product Sets in Groups
}

\author{
Giorgis Petridis
}

\begin{abstract}
We present a new method to bound the cardinality of triple product sets in groups and give three applications. A new and unexpectedly short proof of the Plünnecke-Ruzsa sumset inequalities for Abelian groups. A new proof of a theorem of Tao on triple products, which generalises these inequalities when no assumption on commutativity is made. A further generalisation of the PlünneckeRuzsa inequalities in general groups.
\end{abstract}

\section{Introduction}

Bounding the cardinality of sumsets is a central problem in additive number theory and has many important applications. The first bounds were obtained by Helmut Plünnecke over forty years ago in [6, 7]. Plünnecke was interested in the integers, but his graph-theoretic method works equally well in any Abelian group. The upper bound he obtained is still the standard:

Theorem 1.1 (Plünnecke). Let $A$ and $B$ be finite sets in an Abelian group. Suppose

$$
|A+B| \leq \alpha|A|
$$

Then there exists $X \subseteq A$ such that

$$
|X+h B| \leq \alpha^{h}|X|
$$

In particular $|A+A| \leq \alpha|A|$ implies $|h A| \leq \alpha^{h}|A|$. 
The bound is sharp in terms of $\alpha$ and $|X|$. Let for example $A$ be a subgroup and $B$ a collection of generic points lying in distinct cosets of $A$. Then $|A+B|=|A||B|$ and so $\alpha=|B|$. On the other hand $|A+h B|=\left(\begin{array}{c}|B|+h-1 \\ h\end{array}\right)|X|$ for all $X \subseteq A$.

Imre Ruzsa, who rediscovered Theorem 1.1 [9, 10, 4], extended Plünnecke's result to sum-and-difference sets. Combining Plünnecke's theorem with his 'triangle inequality' (c.f Section 4) Ruzsa showed:

Theorem 1.2 (Ruzsa). Let $A$ and $B$ be finite sets in an Abelian group. Suppose that $|A+B| \leq \alpha|A|$. Then

$$
|k B-l B| \leq \alpha^{k+l}|A|
$$

provided that $k+l>1$.

The proofs given by Plünnecke and Ruzsa were graph-theoretic and to even provide a sketch one must start with a series of definitions. Terence Tao obtained a purely combinatorial proof, albeit with slightly worse bounds [2, 16]. Under the assumption that $|A+A| \leq \alpha|A|$ he demonstrated

$$
|k A-l A| \leq \alpha^{6(k+l)}|A|
$$

The bound may no longer be sharp in $\alpha$, but the difference is not significant enough to affect applications.

In recent years the study of product sets in not necessarily Abelian groups has gained popularity. The lack of commutativity imposes further restrictions, which make the general outlook quite different. For example Theorem 1.1 no longer holds. A well known counter example is $A=H \cup\{x\}$, where $H$ is a subgroup and $x$ is such that $|H x H|=|H|^{2}$. Then $|A A| \leq 3|A|$, while $|A A A| \geq(|A|-1)^{2}$. Ruzsa was probably the first to realise [12] that an extra condition is necessary should one aspire to extend Theorem 1.1 to the non-Abelian case. In addition to $|A B| \leq \alpha|A|$ one has to at least assume that

$$
|A b B| \leq \alpha|A|
$$

for all $b \in B$. Tao generalised the above results to product sets for the important special case when $A=B[15]$.

Theorem 1.3 (Tao). Let $B$ be a finite set in a group. Suppose that $|B b B| \leq \alpha|B|$ for all $b \in B$ and also that $|B B| \leq \alpha|B|$. Then

$$
\left|B^{h}\right| \leq \alpha^{c h}|B|
$$


for some absolute constant $c$.

The constant is large and is not calculated in [15]. Ruzsa asked in [14] for an explicit value of $c$ to be calculated. It is well known [3, 14, 15] that it is adequate to obtain a bound of the form $|B B B| \leq \alpha^{c / 2}|B|$.

Ruzsa proved a different extension to Theorem 1.1 for $h=2$ by changing the order of multiplication and focusing on $B A B$. By a clever application of Plünnecke's graphtheoretic method he showed in [13] the following.

Theorem 1.4 (Ruzsa). Let $A, B$ and $C$ be finite sets in a group. Suppose that $|A B| \leq$ $\alpha_{1}|A|$ and that $|C A| \leq \alpha_{2}|A|$. Then there exists $X \subseteq A$ such that

$$
|C X B| \leq \alpha_{1} \alpha_{2}|X|
$$

The example given above indicates that Theorem 1.4 does not lead to Plünnecke-type bounds on the cardinality of higher product sets $\left|A B^{h}\right|$.

A more thorough discussion of product set estimates in non-Abelian groups can be found in [14, 15]. In this note we introduce a new and simpler method to obtain (variations of) the above mentioned results.

In Section 2 we establish a variant of Ruzsa's theorem.

Theorem 1.5. Let $A$ and $B$ be finite sets in a group. Suppose that $|A B| \leq \alpha|A|$. Then there exists $X \subseteq A$ such that for all finite sets $C$

$$
|C X B| \leq \alpha|C X|
$$

Theorem 1.5 is not as efficient as Theorem 1.4. It only gives $|C X B| \leq \alpha_{1} \alpha_{2}|A|$. In practice however one often bounds $|X|$ by $|A|$ and so the two statements become equivalent. The advantage in using Theorem 1.5 is that the same $X$ works for all $C$, which helps in some applications. Theorem 1.5 furthermore has an unexpectedly short proof despite the fact that we are multiplying non-identical sets in a not necessarily Abelian group.

In Section 3 we deduce Theorems 1.1 and 1.2 from Theorem 1.5. The deduction is swift and we thus present a short, elementary and entirely self-contained proof, which results to the best known bounds. It should also be noted that, by deducing Theorem 1.1 from Theorem 1.5, we reverse the usual order of doing things. 
In Sections 4 and 5 we study non-Abelian analogues of Theorem 1.1. In section 4 we materialise Ruzsa's suggestion and prove an explicit form of Tao's theorem:

Theorem 1.6. Let $B$ be finite a set in a group. Suppose that $|B B| \leq \alpha|B|$ and $|B b B| \leq \beta|B|$ for all $b \in B$. Then for all $h>2$

$$
\left|B^{h}\right| \leq \alpha^{8 h-17} \beta^{h-2}|B|
$$

$c$ in Theorem 1.3 can thus be taken to be nine. Our approach is inspired by Tao's paper, but by using Theorem 1.5 we get a better dependence on $\alpha, \beta$.

It is well know that a similar approach works in a more general setting. Under the further assumption that $A$ and $B$ have comparable sizes we establish in Section 5 a further generalisation of Theorem 1.1 to the non-Abelian setting.

Theorem 1.7. Let $A$ and $B$ be finite sets in a group. Suppose that

(1) $|A B| \leq \alpha|A|$.

(2) $|A b B| \leq \beta|A|$ for all $b \in B$.

(3) $|A| \leq \gamma|B|$.

Then there exists $S \subseteq A$ such that for all $h>1$

$$
\left|S B^{h}\right| \leq \alpha^{8 h-9} \beta^{h-1} \gamma^{4 h-5}|S|
$$

Acknowledgement. The author would like to thank Tim Gowers for his suggestion to look at the non-Abelian setting and other recommendations that improved the presentation of this note.

\section{Growth of Triple Products}

Our method is based on the choice of the subset $X$. We chose it to be a subset of $A$ that grows minimally under multiplication by $B$. The motivation for doing this comes from Plünnecke's original graph-theoretic method. A more illuminative explanation of why this is a natural choice can be found in [1]. More specifically in the first comment by Tim Gowers. 
Let us begin by explaining what we mean by minimal growth under multiplication by $B$. For any $Z \subseteq A$ we define the quantity

$$
r(Z)=\frac{|Z B|}{|Z|}
$$

We let

$$
K=\min _{Z \subseteq A} r(Z)
$$

so that $|Z B| \geq K|Z|$ for all $Z \subseteq A$. We choose $X$ to be such that $r(X)=K$. With this in mind we prove a slightly more technical result.

Proposition 2.1. Let $X$ and $B$ be finite sets in a group. Suppose that

$$
K:=\frac{|X B|}{|X|} \leq \frac{|Z B|}{|Z|}
$$

for all $Z \subseteq X$. Then for all finite sets $C$

$$
|C X B| \leq K|C X|=\frac{|C X||X B|}{|X|} .
$$

Proof. Let $C=\left\{c_{1}, \ldots, c_{r}\right\}$. Using this (arbitrary) order of the elements of $C$ we write

$$
C X=\bigcup_{i=1}^{r}\left(c_{i} X_{i}\right)
$$

where $X_{1}=X$ and for $i>1$

$$
X_{i}=\left\{x \in X: c_{i} x \notin\left\{c_{1}, \ldots, c_{i-1}\right\} X\right\}
$$

Observe that for all $j$ :

$$
\left\{c_{1}, \ldots, c_{j}\right\} X=\bigcup_{i=1}^{j}\left(c_{i} X\right)=\bigcup_{i=1}^{j}\left(c_{i} X_{i}\right) .
$$

The sets $c_{i} X_{i}$ are disjoint and so for all $j$ :

$$
\left|\left\{c_{1}, \ldots, c_{j}\right\} X\right|=\sum_{i=1}^{j}\left|c_{i} X_{i}\right|=\sum_{i=1}^{j}\left|X_{i}\right| .
$$

We proceed by induction on $r$. For $r=1$ we have $\left|c_{1} X B\right|=|X B|=K|X|=K\left|c_{1} X\right|$. For $r>1$ let us write $X_{r}^{c}=X \backslash X_{r}$ for the complement of $X_{r}$ in $X$. By the definition of 
$X_{r}$ we have that $c_{r} X_{r}^{c} \subseteq\left\{c_{1}, \ldots, c_{r-1}\right\} X$ and thus $c_{r} X_{r}^{c} B \subseteq\left\{c_{1}, \ldots, c_{r-1}\right\} X B$. Hence

$$
C X B=\left\{c_{1}, \ldots, c_{r}\right\} X B \subseteq\left(\left\{c_{1}, \ldots, c_{r-1}\right\} X B\right) \cup\left(\left(c_{r} X B\right) \backslash\left(c_{r} X_{r}^{c} B\right)\right)
$$

Note that $\left|\left(c_{r} X B\right) \backslash\left(c_{r} X_{r}^{c} B\right)\right|=\left|(X B) \backslash\left(X_{r}^{c} B\right)\right|=|X B|-\left|X_{r}^{c} B\right|$ and so in particular

$$
|C X B| \leq\left|\left\{c_{1}, \ldots, c_{r-1}\right\} X B\right|+\left(|X B|-\left|X_{r}^{c} B\right|\right) .
$$

The first summand in the expression (21) above is bounded by the inductive hypothesis on $r$ and (11):

$$
\left|\left\{c_{1}, \ldots, c_{r-1}\right\} X B\right| \leq K\left|\left\{c_{1}, \ldots, c_{r-1}\right\} X\right|=K \sum_{i=1}^{r-1}\left|X_{i}\right| .
$$

The second bracketed term in (2) is at most $K\left|X_{r}\right|$ as

$$
\begin{aligned}
|X B|-\left|X_{r}^{c} B\right| & \leq K|X|-K\left|X_{r}^{c}\right| \\
& =K\left(|X|-\left|X_{r}^{c}\right|\right) \\
& =K\left|X_{r}\right|
\end{aligned}
$$

the inequality following from the condition in the statement of the proposition. Adding these upper bounds for the two terms in (21) gives

$$
|C X B| \leq K \sum_{i=1}^{r}\left|X_{i}\right|
$$

and the proposition follows by (11).

Proposition 2.1 is best possible even in the Abelian case as we see by taking $C, X$ and $B$ to be groups thought of as sets in the Cartesian product $C \times X \times B$. It should also be noted that, as Ruzsa observed in [13], Proposition 2.1] is a somewhat commutative result with associativity playing a crucial role. Theorem 1.5 follows immediately:

Proof of Theorem 1.5. We choose $X \subseteq A$ such that

$$
\frac{|X B|}{|X|} \leq \frac{|Z B|}{|Z|}
$$


for all $Z \subseteq A$ and apply Proposition 2.1 observing that

$$
K=\frac{|X B|}{|X|} \leq \frac{|A B|}{|A|} \leq \alpha .
$$

\section{Plünnecke-Ruzsa Inequalities}

We now apply Proposition 2.1 repeatedly to deduce a slightly stronger version of Theorem 1.1 where the subset $X$ is the same for all $h$. Commutativity is crucial, but in a subtle way.

Theorem 3.1. Let $A$ and $B$ be finite sets in an Abelian group. Suppose that

$$
|A+B| \leq \alpha|A|
$$

Then there exists $X \subseteq A$ such that

$$
|X+h B| \leq \alpha^{h}|X|
$$

holds for all $h$.

Proof. This is done by induction on $h$. Let $X \subseteq A$ be such that

$$
\frac{|X+B|}{|X|} \leq \frac{|Z+B|}{|Z|}
$$

for all $Z \subseteq A$. For $h=1$ simply observe $|X+B| \leq|X||A+B| /|A| \leq \alpha|X|$. For $h>1$ we let $C=(h-1) B$. The condition in the statement of Proposition 2.1 is satisfied and thus

$$
|X+h B|=|(h-1) B+X+B| \leq \alpha|X+(h-1) B| \leq \alpha^{h}|X|
$$

There are circumstances where distinguishing between $|X+B| /|X|$ and $\alpha$ is worthwhile (e.g. in [5]). In most cases however taking $K=\alpha$, like we implicitly did, is adequate. One can strengthen Plünnecke's graph-theoretic inequality along the lines of Theorem 3.1. Details and applications can be found in [5].

Using this stronger form of Theorem 1.1 simplifies slightly the proof of Theorem 1.2. 
Proof of Theorem 1.2. We apply the triangle inequality of Ruzsa 8] that will also be used in the next section. Let $X, Y$ and $Z$ be finite sets in an Abelian group. Then

$$
|X||Y-Z| \leq|X+Y||X+Z|
$$

Setting $X=X, Y=k B$ and $Z=l B$ gives

$$
|X||k B-l B| \leq|X+k B||X+l B| \leq \alpha^{k+l}|X|^{2} \leq \alpha^{k+l}|X||A|
$$

In the traditional deduction it is not enough to apply Theorem 3 , but rather the graphtheoretic inequality from which it follows.

\section{The Non-Abelian Setting: Tao's Theorem}

We now turn to the non-Abelian case and prove Theorem 1.6. The material in this section is similar to Tao's argument in [15]. A key difference is that we use Proposition 2.1. which should be thought of as a non-Abelian analogue of Theorem 1.1. This simplifies the argument and also results in a better dependence on $\alpha, \beta$.

The proofs require two results of Ruzsa. Ruzsa's covering lemma [1]:

Lemma 4.1 (Ruzsa). Let $A$ and $B$ be finite sets in a group. Suppose that $|A B| \leq K|A|$. Then there exists a set $S \subseteq B$ of size at most $K$ such that $B \subseteq A^{-1} A S$.

and Ruzsa's triangle inequality [8], a non-Abelian generalisation of (3) [3, 15] :

Lemma 4.2 (Ruzsa). Let $X, Y$ and $Z$ be finite sets in a group. Then

$$
|X||Y Z| \leq\left|Y X^{-1}\right||X Z|
$$

In both [14, 15] it is shown how the above lemma allows one to pass from an upper bound on triple products to upper bounds on higher products. So in principle it is adequate to get a Plünnecke-type bound for $|B B B|$. In practice it is a little more efficient to combine Lemma 4.2 with Proposition [2.1. We will need the following calculation in both steps of the argument.

Corollary 4.3. Let $A$ and $B$ be finite sets in a group. Suppose that $|B B| \leq \alpha|B|$ and that $|B A B| \leq \alpha^{2}|B|$. Then $\left|B A^{-1} A B^{-1}\right| \leq \alpha^{6}|B|$. 
Proof. We apply Lemma 4.2 with $X=B, Y=B A^{-1}$ and $Z=A B^{-1}$ and get

$$
\left|B A^{-1} A B^{-1}\right| \leq \frac{\left|B A^{-1} B^{-1}\right|\left|B A B^{-1}\right|}{|B|}=\frac{\left|B A B^{-1}\right|^{2}}{|B|} .
$$

To bound $B A B^{-1}$ we once again apply Lemma 4.2. This time we set $X=B^{-1}$, $Y=B A$ and $Z=B^{-1}$

$$
\left|B A B^{-1}\right| \leq \frac{|B A B|\left|B^{-1} B^{-1}\right|}{\left|B^{-1}\right|}=\frac{|B A B||B B|}{|B|} \leq \alpha^{3}|B| .
$$

Putting (44) and (5) together finishes the proof.

We now prove that sets which satisfy the conditions of Theorem 4.4 have small tripling.

Theorem 4.4. Let $B$ be a finite set in a group. Suppose that $|B B| \leq \alpha|B|$ and that $|B b B| \leq \beta|B|$ for all $b \in B$. Then

$$
|B B B| \leq \alpha^{7} \beta|B|
$$

The strategy of the proof is to first choose $A \subseteq B$ that grows minimally under multiplication by $B$. Then use Lemma 4.1 to cover the middle copy of $B$ by $A^{-1} A X$. Finally apply Lemma 4.2 repeatedly to break down the product in terms that are easily bound.

Proof of theorem 4.4. We select $A \subseteq B$ such that

$$
K:=\frac{|A B|}{|A|} \leq \frac{|Z B|}{|Z|}
$$

for all $Z \subseteq B$ and observe that $K \leq \alpha$. Applying Proposition 2.1 for $C=B$ gives

$$
|B A B| \leq K|B A| \leq \alpha|B B| \leq \alpha^{2}|B|
$$

Next we apply Lemma 4.1 and get $T \subseteq B$ of size at most $\alpha$ such that $B \subseteq A^{-1} A T$. In particular we have

$$
B B B \subseteq B A^{-1} A T B .
$$

By setting $X=B, Y=B A^{-1} A$ and $Z=T B$ in Lemma 4.2 we get

$$
|B||B B B| \leq|B|\left|B A^{-1} A T B\right| \leq\left|B A^{-1} A B^{-1}\right||B T B| .
$$


To bound the second term in (77) we use the second condition on the theorem together with the fact that $T \subseteq B$.

$$
|B T B|=\left|\bigcup_{t \in T}(B t B)\right| \leq \sum_{t \in T}|B t B| \leq|T| \beta|B| \leq \alpha \beta|B| .
$$

The first term in (7) is at most $\alpha^{6}|B|$ as we see by combining (61) and Corollary 4.3 , Substituting this in (77) and using (8) gives the desired bound on $|B B B|$.

A slightly weaker bound for $|B B B|$ can be obtained using Theorem 1.4. In this case we do not know whether $|A B| \leq \alpha|A|$ and so have to content with $|A B| \leq|B A B| \leq \alpha^{2}|A|$ - the first inequality following from the fact that for any $b \in B$ we have $|A B|=|b A B|$. This leads to $|T| \leq \alpha^{2}$ and we therefore only get $|B B B| \leq \alpha^{9} \beta$.

Theorem 1.6 is completed by induction on $h$.

Proof of Theorem 1.6. Assume that $h>2$ and select $A \subseteq B$ such that

$$
K:=\frac{|A B|}{|A|} \leq \frac{|Z B|}{|Z|}
$$

for all $Z \subseteq B$ and observe that $K \leq \alpha$. Applying Proposition 2.1 for $C=B$ gives (6). Lemma 4.1 implies that there exists $T \subseteq B$ of size at most $\alpha$ such that $B \subseteq A^{-1} A T$. In particular

$$
B^{h} \subseteq B A^{-1} A T B^{h-2}
$$

By setting $X=B, Y=B A^{-1} A$ and $Z=T B^{h-2}$ we get

$$
\left|B^{h}\right| \leq\left|B A^{-1} A T B^{h-2}\right| \leq \frac{\left|B A^{-1} A B^{-1}\right|}{|B|}\left|B T B^{h-2}\right| \leq \alpha^{6}\left|B T B^{h-2}\right| .
$$

The final inequality comes from (66) and Corollary 4.3. To bound $\left|B T B^{h-2}\right|$ we apply Lemma 4.2 with $X=B^{-1}, Y=B T$ and $Z=B^{h-2}$.

$$
\left|B T B^{h-2}\right| \leq \frac{|B T B|}{\left|B^{-1}\right|}\left|B^{-1} B^{h-2}\right| \leq \alpha \beta\left|B^{-1} B^{h-2}\right| .
$$

The second inequality comes from (8) . To bound $\left|B^{-1} B^{h-2}\right|$ we once again resort to Lemma 4.2 this time setting $X=B, Y=B-1$ and $Z=B^{h-2}$.

$$
\left|B^{-1} B^{h-2}\right| \leq \frac{\left|B^{-1} B^{-1}\right|}{|B|}\left|B^{h-1}\right| \leq \frac{|B B|}{|B|}\left|B^{h-1}\right| \leq \alpha\left|B^{h-1}\right| .
$$


Putting (9), (10) and (11) gives

$$
\left|B^{h}\right| \leq \alpha^{8} \beta\left|B^{h-1}\right|
$$

and the desired bound on $\left|B^{h}\right|$ follows by the inductive hypothesis.

As Ruzsa observed in [14] Theorem 4.4 leads, via a slightly different application of Lemma 4.2, to:

Theorem 4.5. Let $B$ be a finite set in a group and let $\epsilon_{1}, \ldots, \epsilon_{h} \in\{+1,-1\}$. Suppose that $|B B| \leq \alpha|B|$ and $|B b B| \leq \beta|B|$ for all $b \in B$. Then

$$
\left|B B^{\epsilon_{1}} \cdots B^{\epsilon_{h}} B^{-1}\right| \leq\left(\alpha^{7} \beta\right)^{2 h}|B|
$$

\section{The Non-Abelian Setting: Proof of Theorem 1.7}

Theorem 1.7 is proved similarly. We begin with the result corresponding to Corollary 4.3 .

Corollary 5.1. Let $S$ and $B$ be finite sets in a group. Suppose that $|C S B| \leq \alpha|C S|$ for all finite sets $C$ in the group. Then

$$
\left|S S^{-1} S S^{-1}\right| \leq \alpha^{6}\left(\frac{|S|}{|B|}\right)^{3}|S|
$$

Proof. We begin by taking $X=B^{-1}, Y=S S^{-1} S$ and $Z=S^{-1}$ in Lemma 4.2

$$
\left|S S^{-1} S S^{-1}\right| \leq \frac{\left|S S^{-1} S B\right|\left|B^{-1} S^{-1}\right|}{\left|B^{-1}\right|} \leq \frac{\alpha\left|S S^{-1} S\right||S B|}{|B|} \leq \alpha \frac{\left|S S^{-1} S\right| \alpha|S|}{|B|}=\alpha^{2} \frac{\left|S S^{-1} S\right||S|}{|B|} .
$$

The inequalities follow from the condition in the statement of the corollary. For example $|S B|=|s S B|$ for any $s \in S$ and hence $|S B| \leq \alpha|s S|=\alpha|S|$. To bound $\left|S S^{-1} S\right|$ we apply Lemma 4.2 with $X=B^{-1}, Y=S$ and $Z=S^{-1} S$.

$$
\left|S S^{-1} S\right| \leq \frac{|S B|\left|B^{-1} S^{-1} S\right|}{\left|B^{-1}\right|} \leq \frac{\alpha|S|\left|S^{-1} S B\right|}{|B|} \leq \alpha \frac{|S| \alpha\left|S^{-1} S\right|}{|B|}=\alpha^{2} \frac{|S|\left|S S^{-1}\right|}{|B|} .
$$

We are finally left with bounding $S S^{-1}$. We once again apply Lemma 4.2 with $X=$ $B^{-1}, Y=S$, and $Z=S^{-1}$.

$$
\left|S S^{-1}\right| \leq \frac{|S B|\left|B^{-1} S^{-1}\right|}{\left|B^{-1}\right|}=\frac{|S B|^{2}}{|B|} \leq \alpha^{2} \frac{|S|^{2}}{|B|} .
$$


Putting everything together gives the desired bound.

We next show that for any $S \subseteq A$, which grows minimally under multiplication by $B$, $S B B$ satisfies a Plünnecke-type bound.

Proposition 5.2. Let $A$ and $B$ be sets in a finite group. Suppose that

(1) $|A B| \leq \alpha|A|$.

(2) $|A b B| \leq \beta|A|$ for all $b \in B$.

(3) $|A| \leq \gamma|B|$.

Let $S \subseteq A$ be such that

$$
\frac{|S B|}{|S|} \leq \frac{|Z B|}{|Z|}
$$

for all $Z \subseteq A$. Then

$$
|S B B| \leq \alpha^{7} \beta \gamma^{3}|S|
$$

Proof. We begin by observing that Proposition 2.1 can be applied to $S$ and $B$ and so

$$
|C S B| \leq \alpha|C S|
$$

In particular $|S B| \leq \alpha|S|$ and so by Lemma 4.1 there exists $T \subseteq B$ of size at most $\alpha$ such that $B \subseteq S^{-1} S T$. Thus $|S B B| \leq\left|S S^{-1} S T B\right|$. Applying Lemma 4.2 with $X=S$, $Y=S S^{-1} S$ and $Z=T B$ gives

$$
|S B B| \leq\left|S S^{-1} S T B\right| \leq \frac{\left|S S^{-1} S S^{-1}\right|}{|S|}|S T B|
$$

By (12) and Corollary 5.1 we know that the first term in (13) is at most $\alpha^{6} \gamma^{2}|S| /|B|$. The second term in (13) is bounded using the second condition and the fact that $S \subseteq A$ and $T \subseteq B$.

$$
|S T B|=\left|\bigcup_{t \in T}(S t B)\right| \leq \sum_{t \in T}|S t B| \leq \sum_{t \in T}|A t B| \leq|T| \beta|A| \leq \alpha \beta|A| .
$$

Substituting in (13) gives

$$
|S B B| \leq \alpha^{6} \gamma^{2} \frac{|S|}{|B|} \alpha \beta|A| \leq \alpha^{7} \beta \gamma^{3}|S| .
$$


Theorem 1.7 follows by induction on $h$.

Proof of Theorem 1.7. We work with $S \subseteq A$ defined in the statement of Proposition 5.2. Proposition 2.1 implies that (12) holds. Next we apply Lemma 4.1 and get $T \subseteq B$ of size at most $\alpha$ such that $B \subseteq A^{-1} A T$. In particular

$$
\left|S B^{h}\right| \subseteq\left|S S^{-1} S T B^{h-1}\right|
$$

By setting $X=S, Y=S S^{-1} S$ and $Z=T B^{h-1}$ in Lemma 4.2 we get

$$
\left|S B^{h}\right| \leq \frac{\left|S S^{-1} S S^{-1}\right|}{|S|}\left|S T B^{h-1}\right|
$$

The second term is bounded by applying Lemma 4.2 with $X=B^{-1}, Y=S T$ and $Z=B^{h-1}$.

$$
\left|S T B^{h-1}\right| \leq \frac{|S T B|\left|B^{-1} B^{h-1}\right|}{\left|B^{-1}\right|} \leq \alpha \beta \gamma\left|B^{-1} B^{h-1}\right| .
$$

The second inequality follows from (14). We are thus left to bound $\left|B^{-1} B^{h-1}\right|$. Setting $X=S, Y=B^{-1}$ and $Z=B^{h-1}$ in Lemma 4.2 gives

$$
\left|B^{-1} B^{h-1}\right| \leq \frac{\left|B^{-1} S^{-1}\right|\left|S B^{h-1}\right|}{|S|}=\frac{|S B|}{|S|}\left|S B^{h-1}\right| \leq \alpha\left|S B^{h-1}\right| .
$$

Substituting (16) and (17) in (15) and applying Corollary 5.1 we get

$$
\left|S B^{h}\right| \leq \alpha^{6} \gamma^{3} \alpha \beta \gamma \alpha\left|S B^{h-1}\right|=\alpha^{8} \beta \gamma^{4}\left|S B^{h-1}\right|
$$

and the theorem follows from the inductive hypothesis.

\section{References}

[1] W.T. Gowers. A new way of proving sumset estimates. gowers.wordpress.com/2011/02/10/a-new-way-of-proving-sumset-estimates/.

[2] A Granville. An introduction to additive combinatorics. In A. Granville, M. B Nathanson, and J. Solymosi, editors, Additive Combinatorics, CRM Proceedings \& Lecture Notes, pages 1-27, New York, 2006. American Mathamatical Society. 
[3] H. A. Helfgott. Growth and generation in $S L_{2}(Z / p Z)$. Ann. of Math., 167:601$623,2008$.

[4] J. L. Malouf. On a theorem of Plünnecke concerning the sum of a basis and a set of positive density. J. Number Theory, 54:12-22, 1995.

[5] G. Petridis. Upper bounds on the cardinality of higher sumsets. math.co/1101.5001. Submitted.

[6] H. Plünnecke. Eigenschaften und Abschätzungen von Wirkungsfunktionen. Berichte der Gasellschaft für Mathematik und Datenverarbeitung, Bonn, 1969.

[7] H. Plünnecke. Eine zahlentheoretische anwendung der graphtheorie. J. Reine Angew. Math., 243:171-183, 1970.

[8] I. Z. Ruzsa. On the cardinality of $A+A$ and $A-A$. In Combinatorics (Keszthely 1976) Coll. Math. Soc. J. Bolyai, vol 18, pages 933-938, Budapest, 1978. NorthHolland - Bolyai Társulat.

[9] I. Z. Ruzsa. An application of graph theory to additive number theory. Scientia, Ser. A, 3:97-109, 1989.

[10] I. Z. Ruzsa. Addendum to: An application of graph theory to additive number theory. Scientia, Ser. A, 4:93-94, 1990/1991.

[11] I. Z. Ruzsa. An analogue of Freiman's theorem in groups. Asterisque, 258:323-326, 1999.

[12] I. Z. Ruzsa. Cardinality questions about sumsets. In A. Granville, M. B Nathanson, and J. Solymosi, editors, Additive Combinatorics, CRM Proceedings \& Lecture Notes, pages 195-204, New York, 2006. American Mathamatical Society.

[13] I. Z. Ruzsa. Sumsets and structure. In Combinatorial Number Theory and Additive Group Theory. Springer, New York, 2009.

[14] I. Z. Ruzsa. Towards a noncommutative Plünnecke-type inequality. In I. Bárány and J. Solymosi, editors, An Irregular Mind Szemeredi is 70, Bolyai Society Mathematical Studies, Vol. 21, New York, 2010. Springer.

[15] T. Tao. Product set estimates for non-commutative groups. Combinatorica, 28(5):547-594, 2008. 
[16] T. Tao and V.H. Vu. Additive Combinatorics. Cambridge University Press, Cambridge, 2006.

Department of Pure Mathematics and Mathematical Statistics Wilberforce Road, Cambridge CB3 0WB, England

Email address: giorgis@cantab.net 